\title{
Analysis of the Movement of ICD Leads During Cardiac Contraction as Determinant of Intracardiac Impedance
}

This paper was downloaded from TechRxiv (https://www.techrxiv.org).

\section{LICENSE}

CC BY 4.0

SUBMISSION DATE / POSTED DATE

$10-01-2021 / 13-01-2021$

\section{CITATION}

Kirchner, Jens; Arnold, Martin; Fischer, Georg (2021): Analysis of the Movement of ICD Leads During Cardiac Contraction as Determinant of Intracardiac Impedance. TechRxiv. Preprint. https://doi.org/10.36227/techrxiv.13551395.v1

DOI

10.36227/techrxiv.13551395.v1 


\title{
Analysis of the Movement of ICD Leads During Cardiac Contraction as Determinant of Intracardiac Impedance
}

\author{
Jens Kirchner ${ }^{1}$, Martin Arnold ${ }^{2}$ and Georg Fischer ${ }^{1}$
}

\begin{abstract}
Intracardiac impedance (ICI) has been proposed as an indicator of cardiac status in heart failure patients. We introduce a biophysical model of the measurement setup and apply it to the movement of ICD leads reconstructed from clinical-routine X-ray recordings in a study population of 12 patients. Tilting of the right ventricular lead is found to be a major determinant of ICI changes during cardiac contraction with a mean contribution of $42 \pm 23 \%$. The relative position between right and left ventricular lead is the second major contributor $(40 \pm 22 \%)$. However, the contributions of the components of movement strongly differ between the patients. The proposed method provides means for a better interpretation of ICI measurements and for an improvement of its performance for monitoring heart failure status.
\end{abstract}

\section{INTRODUCTION}

Remote monitoring of cardiovascular diseases has been subject to intensive research in the past years [1]. Particularly patients with implanted cardioverter-defibrillators (ICD) can profit from continuous capture of vital parameters by implanted sensors [2], [3], [4]. In recent years, intracardiac impedance (ICI) has been introduced as an indicator of heart failure status [5], [6]. It denotes a quadrupolar impedance measurement with current injection between tip and shock coil of the right ventricular (RV) lead and voltage measurement between tip and ring of the left ventricular (LV) lead. Baseline and peak-to-peak amplitude during cardiac contraction were proposed as correlates of LV volume and stroke volume, respectively. The diagnostic value of ICI has been positively evaluated in several acute studies [7], [8], [9]. While these studies focus on correlations between ICI parameters and hemodynamic quantities, the underlying biophysical model has not been investigated up to now. Therefore, we here provide the description of ICI as the result of the LV lead moving in the electric dipole field generated by the RV lead. This model is combined with a recently proposed method to reconstruct the 3-dimensional movement of ICD-leads from X-ray movies as recorded as part of clinical routine during implantation [10]. In this way, this large data pool of X-ray recordings can be used to investigate lead movement and its impact on ICI in detail.

In the following, this approach will be applied to a study population of 12 patients. The movement of the ICD leads will be split into several physiologically meaningful

\footnotetext{
${ }^{1}$ Jens Kirchner and Georg Fischer are with Institute for Electronics Engineering, Friedrich-Alexander-University Erlangen-Nuernberg (FAU), 91058 Erlangen, Germany (e-mail: jens.kirchner@fau.de).

${ }^{2}$ Martin Arnold is with Medical Clinic 2, Cardiology and Angiology, University Hospital Erlangen, 91058 Erlangen, Germany.
}

components in order to identify the dominant influencing factors of ICI.

\section{METHODS}

\section{A. Data acquisition}

$\mathrm{X}$-ray movies recorded as standard practice during implantation at University Hospital Erlangen, Germany, were used. All movies were captured with an Artis zee (Siemens Healthcare, Erlangen, Germany). A movie was usable for analysis if it had a temporal resolution of at least 15 frames per second and an ECG that was applicable for synchronization and if it contained a time interval of at least one heart cycle with all relevant parts of the leads visible. Data from a patient were evaluated if two usable movies from two perspectives differing by at least $20^{\circ}$ were available.

\section{B. Reconstruction of 3D-movement}

The reconstruction of the 3-dimensional movement of the ICD leads from the available X-ray data has recently been described in [10]: The two successively recorded (2dimensional) X-ray movies were synchronized with respect to the cardiac cycle and, if possible, to respiration. They were then treated as synchronous recordings in order to obtain the $3 \mathrm{D}$-coordinates from the intersection of the projection lines. From that, five vectors $\vec{\xi}_{X}$ are obtained that give the loci of the relevant points $X$ over time: the distal and proximal end of the right ventricular shock coil ( $X=$ 'RVcoilD' and 'RVcoilP', respectively), the tip of the right ventricular lead ('RVtip') as well as ring and tip of the left ventricular lead ('LVring' and 'LVtip', respectively).

Reconstruction quality was assessed in terms of deviation from an exact intersection of the projection lines and of deviation from a known reference length: Patients were discarded if the upper quartile of the minimum distances between projection lines exceeded $5 \mathrm{~mm}$ and if the reconstructed distance between proximal and distal end of the ICD shock coil exceeded the length of $5 \mathrm{~cm}$ by more than $2.5 \mathrm{~mm}$.

\section{Components of movement}

For calculating intracardiac impedance, only the loci of the four poles (RVcoilD, RVtip, LVring and LVtip) are relevant and will be considered in the following. In order to interpret their 3-dimensional movement and the resulting impedance in a physiologically meaningful way, the a center-of-mass 
vector $\vec{c}$ and a directional vector $\vec{d}$ were derived for each lead:

$$
\begin{aligned}
\vec{c}_{\mathrm{RV}} & =\frac{\vec{\xi}_{\text {RVcoilD }}+\vec{\xi}_{\text {RVtip }}}{2} \\
\vec{d}_{\mathrm{RV}} & =\xi_{\text {RVtip }}-\xi_{\text {RVcoild }} \\
\vec{c}_{\mathrm{LV}} & =\frac{\vec{\xi}_{\text {RVcoilD }}+\vec{\xi}_{\text {RVtip }}}{2} \\
\vec{d}_{\mathrm{LV}} & =\xi_{\text {RVtip }}-\xi_{\text {RVcoilD }} .
\end{aligned}
$$

Furthermore, the center of mass of both leads

$$
\vec{C}_{\mathrm{RVLV}}=\frac{\vec{c}_{\mathrm{RV}}+\vec{c}_{\mathrm{LV}}}{2}
$$

and the connecting vector between the two ventricles

$$
\vec{D}_{\mathrm{RVLV}}=\vec{c}_{\mathrm{LV}}-\vec{c}_{\mathrm{RV}}
$$

were defined.

With the relationships above the set of the four poles

$$
\left(\vec{\xi}_{\text {RVcoilD }}, \vec{\xi}_{\text {RVtip }}, \vec{\xi}_{\text {LVring }}, \vec{\xi}_{\text {LVtip }}\right)
$$

can be reformulated to more meaningful sets of independent components of movement. The first approach uses the centerof-mass and directional vector of each lead and thus takes the two ventricles as independent of each other:

$$
\left(\vec{c}_{\mathrm{RV}}, \vec{d}_{\mathrm{RV}}, \vec{c}_{\mathrm{LV}}, \vec{d}_{\mathrm{LV}}\right)
$$

In contrast, the second approach focuses on the movement of the ventricles relative to each other:

$$
\left(\vec{C}_{\mathrm{RVLV}}, \vec{D}_{\mathrm{RVLV}}, \vec{d}_{\mathrm{RV}}, \vec{d}_{\mathrm{LV}}\right)
$$

The two approaches will be termed 'Independent ventricles' and 'Center of mass'.

Note that the directional vectors will affect ICI rather by change of orientation than by a change of length, as the distance between the poles on a lead will not vary much due to the stiffness of the leads.

\section{Intracardiac impedance}

The ICI measurement setup is captured by the physical model of the RV lead acting as a point dipole and the LV lead moving in the corresponding potential, as was briefly indicated in [11] and will be derived in the following.

The values for relative permittivity $\epsilon_{r}$ and conductivity $\sigma$ that are use in the following were taken from the IT'IS database ([12]; see also [13]) for measurement frequency $128 \mathrm{~Hz}$ :

$$
\begin{array}{lll}
\text { Blood: } & \epsilon_{r} \approx 5 \cdot 10^{3} & \sigma \approx 7 \cdot 10^{-1} \\
\text { Myocardium: } & \epsilon_{r} \approx 2 \cdot 10^{6} & \sigma \approx 1 \cdot 10^{-1} \frac{\mathrm{S}}{\mathrm{m}}
\end{array}
$$

With the conductivity of body tissue being much smaller than that of the lead wires, RV coil and tip form a capacitor with capacitance limited by the surface area of the tip electrode with $4.5 \mathrm{~mm}^{2}$, compared to RVcoil with $290 \mathrm{~mm}^{2}$ [14]. With the specific capacitance of a microporous electrode surface of approximately $4 \frac{\mu \mathrm{F}}{\mathrm{mm}^{2}}$ [15] and the RV tip surface stated above, capacitance is estimated to be

$$
C_{\mathrm{RV}} \approx 4.5 \mathrm{~mm}^{2} \cdot 4 \frac{\mu \mathrm{F}}{\mathrm{mm}^{2}}=18 \mu \mathrm{F}
$$

The voltage between the RV poles is derived from the applied current $I_{\mathrm{RV}}=200 \mu \mathrm{A}$ and an estimate of the static resistance between the poles,

$$
R_{\mathrm{RV}}=\frac{1}{\sigma_{\text {blood }}} \frac{d_{\mathrm{RV}}}{A} \approx \frac{1}{7 \cdot 10^{-1} \frac{S}{m}} \frac{17 \mathrm{~mm}}{1 \mathrm{~cm}^{2}} \approx 240 \Omega,
$$

where conductance was assumed mainly via blood over the distance $d_{\mathrm{RV}}=1.7 \mathrm{~cm}$ between RV tip and shock coil (distal end) [14] and through an area of order $1 \mathrm{~cm}^{2}$. Hence, the voltage between the RV tip and coil is

$$
U_{\mathrm{RV}}=R_{\mathrm{RV}} \cdot I_{\mathrm{RV}} \approx 240 \Omega \cdot 200 \mu \mathrm{A} \approx 50 \mathrm{mV}
$$

The resulting negative and positive charges $\mp q_{\mathrm{RV}}$ on RV tip and on the distal end of the shock coil, respectively, hence amount to

$$
q_{\mathrm{RV}}=C_{\mathrm{RV}} \cdot U_{\mathrm{RV}} \approx 18 \mu \mathrm{F} \cdot 50 \mathrm{mV} \approx 9 \cdot 10^{-7} \mathrm{C}
$$

Note that the actual charge will be smaller than this value: The estimation assumes a static picture with a fully charged capacitor, whereas, with the alternating current at $128 \mathrm{~Hz}$ and pulse width $30 \mu \mathrm{s}$, time will not suffice to fully charge, such that even peak charge will not reach the maximum value estimated above.

As the charges are concentrated at the tip and the distal end of the shock coil, they are treated as point-shaped, such that the RV electrode forms an electric dipole with moment

$$
\vec{p}_{\mathrm{RV}}=q_{\mathrm{RV}} \cdot \vec{d}_{\mathrm{RV}}
$$

where vector $\vec{d}_{\mathrm{RV}}$ points from the negative to the positive charge, i. e., from RV tip to the distal end of RV coil.

The dipole potential at some point $\vec{r}$ relative to the center of the dipole is then given as

$$
\varphi(\vec{r})=\frac{q_{\mathrm{RV}}}{4 \pi \epsilon_{0} \epsilon_{r}} \frac{\vec{d}_{\mathrm{RV}} \cdot \vec{r}}{\|\vec{r}\|^{3}},
$$

where the relative permittivity is assumed to be dominated by the damping contribution of the myocardium and hence $\epsilon_{r} \approx 2 \cdot 10^{6}$.

The dipole potential (15) induces a potential difference between $\mathrm{LV}$ tip and ring, i. e., measured voltage $U_{\mathrm{LV}}$. Hence, theoretical intracardiac impedance is

$$
Z=\frac{U_{\mathrm{LV}}}{I_{\mathrm{RV}}}=\frac{1}{I_{\mathrm{RV}}}\left[\varphi\left(\vec{r}_{\mathrm{LVring}}\right)-\varphi\left(\vec{r}_{\mathrm{LVtip}}\right)\right]
$$

\section{E. Contribution of components of movement}

As derived in the previous section, intracardiac impedance (16) depends on the loci of the four poles RV coil (proximal end), RV tip, LV ring and LV tip. It can hence be expressed as a function of one of the three sets of vectors (7), (8) and (9). In order to estimate the contributions of the independent components in each set, a differential approach is taken: The small changes of impedance between successive measurement times are split up into a superposition of impedance changes due each component:

$$
\begin{aligned}
\Delta Z\left(t_{i+1}\right) & =Z\left(\vec{r}_{1}^{\prime}, \vec{r}_{2}^{\prime}, \vec{r}_{3}^{\prime}, \vec{r}_{4}^{\prime}\right)-Z\left(\vec{r}_{1}, \vec{r}_{2}, \vec{r}_{3}, \vec{r}_{4}\right) \\
& =\sum_{k=1}^{4} \Delta Z_{k}\left(t_{i+1}\right)+\epsilon
\end{aligned}
$$


where

$$
\vec{r}_{k}=\vec{r}_{k}\left(t_{i}\right) \quad \text { and } \quad \vec{r}_{k}^{\prime}=\vec{r}_{k}\left(t_{i+1}\right),
$$

$\left(\vec{r}_{1}, \vec{r}_{2}, \vec{r}_{3}, \vec{r}_{4}\right)$ denotes one of the sets of components of movement (8) or (9),

$$
\begin{aligned}
& \Delta Z_{1}\left(t_{i+1}\right)=Z\left(\vec{r}_{1}^{\prime}, \vec{r}_{2}, \vec{r}_{3}, \vec{r}_{4}\right)-Z\left(\vec{r}_{1}, \vec{r}_{2}, \vec{r}_{3}, \vec{r}_{4}\right) \\
& \Delta Z_{2}\left(t_{i+1}\right)=Z\left(\vec{r}_{1}, \vec{r}_{2}^{\prime}, \vec{r}_{3}, \vec{r}_{4}\right)-Z\left(\vec{r}_{1}, \vec{r}_{2}, \vec{r}_{3}, \vec{r}_{4}\right) \\
& \Delta Z_{3}\left(t_{i+1}\right)=Z\left(\vec{r}_{1}, \vec{r}_{2}, \vec{r}_{3}^{\prime}, \vec{r}_{4}\right)-Z\left(\vec{r}_{1}, \vec{r}_{2}, \vec{r}_{3}, \vec{r}_{4}\right) \\
& \Delta Z_{4}\left(t_{i+1}\right)=Z\left(\vec{r}_{1}, \vec{r}_{2}, \vec{r}_{3}, \vec{r}_{4}^{\prime}\right)-Z\left(\vec{r}_{1}, \vec{r}_{2}, \vec{r}_{3}, \vec{r}_{4}\right)
\end{aligned}
$$

and $\epsilon$ is the deviation of $\Delta Z\left(t_{i+1}\right)$ from a purely linear superposition of the $\Delta Z_{k}\left(t_{i+1}\right)$.

The mean relative contribution $p_{k}$ of a specific component of movement $\vec{r}_{k}$ is then given by the weighted average

$$
p_{k}=\sum_{i} w\left(t_{i}\right) \cdot \frac{\Delta Z_{k}\left(t_{i}\right)}{\Delta Z\left(t_{i}\right)}
$$

where the weighting factor is given by

$$
w\left(t_{i}\right)=\frac{\left\|\Delta Z\left(t_{i}\right)\right\|}{\sum_{i}\left\|\Delta Z\left(t_{i}\right)\right\|} .
$$

\section{RESULTS \& DISCUSSION}

\section{A. Data acquisition and reconstruction quality}

$\mathrm{X}$-ray movies that were usable for data analysis as defined in section II-A were obtained from 16 patients (50\% male, $61 \pm 10$ years old). All movies were recorded with a sampling frequency of $15 \mathrm{fps}$ and contained between one and three heart cycles (14-52 frames). The perspectives between the two movies of each patient differed by $21^{\circ}$ to $58^{\circ}$. The reconstructed 3D-motion provided sufficient quality (see section II-B) in 12 cases.

\section{B. Intracardiac impedance}

Offset (i. e. mean value) and (peak-peak) amplitude of the intracardiac impedance derived from the reconstructed 3Dmovement by use of (16) are given in Tab. I. Offset varies between $-2.5 \Omega$ and $10.3 \Omega$ and amounts to $4.0 \Omega$ in average. The amplitude ranges from 0.9 up to $7.7 \Omega$ and is $2.9 \Omega$ in average.

\section{Contributions of components of movement}

Table II gives the mean relative contributions $p_{k}$ as defined in (24) for the two sets of components of movement (8) ('Independent ventricles') and (9) ('Center of mass').

The error made when splitting impedance changes into different components amounts to $11 \%$ and $10 \%$ for the two vector sets. The approach is hence accurate enough to estimate the dominant contributions.

In both vector sets, the directional vector $\vec{d}_{\mathrm{RV}}$ provides an average contribution of $42 \%$ and is hence a major determinant of intracardiac impedance. This is due to the fact that the right ventricular poles generates the electric dipole potential. A tilting of the RV lead will thus rotate the entire potential and can thus induce large changes of the potential difference sensed at the LV poles.
TABLE I

INTRACARDIAC IMPEDANCE AS DERIVED FROM RECONSTRUCED 3D-MOVEMENT.

\begin{tabular}{ccc}
\hline \hline Patient & Offset $(\Omega)$ & Amplitude $(\Omega)$ \\
\hline $\mathbf{1}$ & 4.8 & 7.7 \\
$\mathbf{2}$ & 6.0 & 1.5 \\
$\mathbf{3}$ & 2.5 & 2.0 \\
$\mathbf{4}$ & 4.2 & 0.9 \\
$\mathbf{5}$ & 9.8 & 6.0 \\
$\mathbf{6}$ & -2.5 & 2.4 \\
$\mathbf{7}$ & 10.3 & 3.5 \\
$\mathbf{8}$ & 1.1 & 1.4 \\
$\mathbf{9}$ & 3.3 & 1.7 \\
$\mathbf{1 0}$ & 1.8 & 2.4 \\
$\mathbf{1 1}$ & 2.5 & 3.3 \\
$\mathbf{1 2}$ & 4.4 & 2.5 \\
\hline Mean \pm Standard deviation & $4.0 \pm 3.5$ & $2.9 \pm 2.0$ \\
\hline \hline
\end{tabular}

With a mean contribution of $18 \%$, the impact of the left ventricular directional vector is considerably smaller than that of the right ventricle. Nevertheless, in certain configurations as with patient no. $6, \vec{d}_{\mathrm{LV}}$ can take the role of the dominant factor and be responsible for up to $90 \%$ of the impedance changes.

While the vector sets (8) and (9) share the directional components $\vec{d}_{\mathrm{RV}}$ and $\vec{d}_{\mathrm{LV}}$, they differ in the remaining components, $\vec{c}_{\mathrm{RV}}, \vec{c}_{\mathrm{LV}}$ and $\vec{C}_{\mathrm{RVLV}}, \vec{D}_{\mathrm{RVLV}}$, respectively. In the latter approach, the center of mass of all four poles, $\vec{C}_{\mathrm{RVLV}}$, does not contribute at all to impedance changes, as it does not affect the position of the poles relative to each other. The connecting vector between RV center and LV center, $\vec{D}_{\mathrm{RVLV}}$, however, is of similar importance as $\vec{d}_{\mathrm{RV}}$ and provides an average contribution of $40 \%$. In patient no. 12 , it even takes the role of the dominant factor and amounts to $62 \%$.

From the $p_{k}$-values of the individual patients it becomes clear that the contributions to ICI changes vary dramatically between the subjects and can considerably deviate from the average values. This is due to two different reasons: First, the movement of the myocardium will differ between the patients, due medical history, genetic factors etc. Second, the position of the leads within the heart depends on implantation and varies between the patients. Due to the strong nonlinearity of the dipole potential, the resulting position of the $\mathrm{LV}$ poles relative to the RV lead can strongly affect lead movement and thus ICI.

\section{Limitations}

It is important to indicate the limitations of the method applied here. First, as mentioned in section II-B, nonsynchronous $\mathrm{X}$-ray recordings were treated as depicting the same cardiac movement. The 3D motion reconstructed from these movies will only be accurate if cardiac contraction is sufficiently similar in both movies. By synchronization to respiration as well as by discarding data with low reconstruction quality, however, measures were taken to reduce the 
TABLE II

MEAN RELATIVE CONTRIBUtions (24) FOR THE SETS OF COMPONENTS OF MOVEMENT (8) ('INDEPENDENT VENTRICLES') AND (9) ('CENTER OF MASS'). CONTRIBUTIONS ARE GIVEN FOR EACH PATIENT AS WELL AS MEAN, STANDARD DEVIATION AND RANGE FOR THE STUDY POPULATION.

\begin{tabular}{|c|c|c|c|c|c|c|c|c|c|c|}
\hline \multirow[t]{2}{*}{ Patient } & \multicolumn{5}{|c|}{ Independent ventricles } & \multicolumn{5}{|c|}{ Center of mass } \\
\hline & $\vec{c}_{\mathrm{RV}}$ & $\vec{c}_{\mathrm{LV}}$ & $\vec{d}_{\mathrm{RV}}$ & $\vec{d}_{\mathrm{LV}}$ & $\|\epsilon\|$ & $\vec{C}_{\mathrm{RVLV}}$ & $\vec{D}_{\mathrm{RVLV}}$ & $\vec{d}_{\mathrm{RV}}$ & $\vec{d}_{\mathrm{LV}}$ & $\|\epsilon\|$ \\
\hline 1 & 17 & 3 & 82 & -3 & 14 & 0 & 19 & 82 & -3 & 13 \\
\hline 2 & 3 & 65 & 51 & -18 & 11 & 0 & 69 & 51 & -18 & 7 \\
\hline 3 & 61 & -2 & 31 & 9 & 9 & 0 & 57 & 31 & 9 & 7 \\
\hline 4 & -8 & 25 & 43 & 41 & 8 & 0 & 17 & 43 & 41 & 8 \\
\hline 5 & 43 & 4 & 24 & 28 & 7 & 0 & 48 & 24 & 28 & 6 \\
\hline 6 & 7 & 0 & 3 & 90 & 6 & 0 & 7 & 3 & 90 & 6 \\
\hline 7 & 2 & 19 & 44 & 28 & 12 & 0 & 21 & 44 & 28 & 12 \\
\hline 8 & 30 & 26 & 29 & 11 & 12 & 0 & 57 & 29 & 11 & 12 \\
\hline 9 & -8 & 71 & 51 & -5 & 21 & 0 & 60 & 51 & -5 & 20 \\
\hline 10 & -8 & 26 & 78 & 7 & 10 & 0 & 16 & 78 & 7 & 9 \\
\hline 11 & 26 & 19 & 46 & 8 & 8 & 0 & 46 & 46 & 8 & 8 \\
\hline 12 & 23 & 39 & 21 & 17 & 8 & 0 & 62 & 21 & 17 & 7 \\
\hline $\begin{array}{c}\text { Mean } \pm \text { Standard deviation } \\
\text { Range }\end{array}$ & $\begin{array}{l}16 \pm 22 \\
-8 \ldots 61\end{array}$ & $\begin{array}{l}25 \pm 24 \\
-2 \ldots 71\end{array}$ & $\begin{array}{c}42 \pm 23 \\
3 \ldots 82\end{array}$ & $\begin{array}{l}18 \pm 28 \\
-18 \ldots 90\end{array}$ & $\begin{array}{c}11 \pm 4 \\
6 \ldots 21\end{array}$ & $\begin{array}{c}0 \pm 0 \\
0 \ldots 0\end{array}$ & $\begin{array}{l}40 \pm 22 \\
7 \ldots 69\end{array}$ & $\begin{array}{c}42 \pm 23 \\
3 \ldots 82\end{array}$ & $\begin{array}{l}18 \pm 28 \\
-18 \ldots 90\end{array}$ & $\begin{array}{c}10 \pm 4 \\
6 \ldots 20\end{array}$ \\
\hline
\end{tabular}

effect of asynchronous X-ray capture as much as possible.

Second, the model captures only the effects of lead movement, while assuming the poles of the impedance measurement being located in a homogenous medium. The effects of changing proportions of blood and myocardium with their specific relative permittivity, however, were not considered. This has to be addressed in future investigations based on detailed imaging data, e.g. from MRI, and FEM simulations.

\section{CONCLUSION}

The results provided above show that intracardiac impedance is a complex sensor quantity that is affected by different components of lead movement during cardiac contraction, e.g. by tilting of the right or left ventricular lead, change of position of the leads relative to each other etc. The contributions of these components of movement vary considerably between different patients. Hence, if ICI is used for diagnostic purposes as desired, interpretation of the data has to be based on the knowledge of what components are responsible for the observed ICI signal. For example, an ICI signal that is dominated by RV tilting cannot be expected to be a sensitive indicator of LV heart failure status.

The method proposed above provides means to derive the relevant components of lead movement from X-ray recordings that are part of clinical routine during implantation. Hence, it can help to better interpret ICI data and thus improve its performance for monitoring heart failure status.

\section{REFERENCES}

[1] P. S. Hong, A. N. Sulke, and Z. R. Yousef, "Remote monitoring of cardiovascular disease: great promise, but do not forget the patient!" Int. J. Clin. Pract., vol. 68, no. 6, pp. 674-678, 2014.

[2] C. Opasich, S. Capomolla, P. G. Riccardi, O. Febo, G. Forni, F. C. $\mathrm{F}$, and L. Tavazzi, "Does in-patient ecg monitoring have an impact on medical care in chronic heart failure patients?" Eur. J. Heart Fail., vol. 2 , no. 3 , pp. $281-285,2000$
[3] L. Yu, B. J. Kim, and E. Meng, "Chronically implanted pressure sensors: Challenges and state of the field," Sensors, vol. 14, no. 11, pp. $20620-20644,2014$.

[4] D. J. van Veldhuisen, F. Braunschweig, V. Conraads, I. Ford, M. R Cowie, G. Jondeau, J. Kautzner, R. M. Aguilera, M. Lunati, C. M. Yu, B. Gerritse, and M. Borggrefe, "Intrathoracic impedance monitoring, audible patient alerts, and outcome in patients with heart failure," Circulation, vol. 124, pp. 1719-1726, 2011.

[5] W. Arthur and G. C. Kaye, "Clinical use of intracardiac impedance: Current applications and future perspectives," Pacing Clin. Electrophysiol., vol. 24, no. 4, pp. 500-506, 2001.

[6] M. Lippert, S. Berdyshev, G. Czygan, M. Bocchiardo, and B. Hensel, "Intracardiac impedance as a method for ventricular volume monitoring - investigation by a finite-element model and clinical data," $J$. Phys. Conf. Ser, vol. 224, no. 1, p. 012095, 2010.

[7] E. Zima, M. Lippert, G. Czygan, and B. Merkely, "Determination of left ventricular volume changes by intracardiac conductance using a biventricular electrode configuration," Europace, vol. 8, no. 7, pp. 537-544, 2006.

[8] M. Bocchiardo, D. Meyer zu Vilsendorf, C. Militello, M. Lippert, G. Czygan, F. Gaita, P. Schauerte, and C. Stellbrink, "Intracardiac impedance monitors stroke volume in resynchronization therapy patients," Europace, vol. 12, no. 5, pp. 702-707, 2010a.

[9] M. Bocchiardo, D. Meyer zu Vilsendorf, C. Militello, M. Lippert, G. Czygan, P. Schauerte, F. Gaita, and C. Stellbrink, "Resynchronization therapy optimization by intracardiac impedance," Europace, vol. 12 , no. 11 , pp. 1589-1595, 2010b.

[10] J. Kirchner, M. Arnold, and G. Fischer, "Reconstruction of 3Dmovement of ICD leads from clinical-routine X-ray movies," in 2017 IEEE Int. Symp. Signal Process. Inf. Technol. (ISSPIT), 2017, to be published.

[11] O. Skerl, M. Lippert, S. Paule, and G. Czygan, "Impedance sensor for assessing cardiac hemodynamics," in IEEE Sensors: 26-29 Oct. 2008, 2008, pp. 1096-1099.

[12] P. A. Hasgall, E. Neufeld, M. C. Gosselin, A. Klingenböck, and N. Kuster, "IT'IS Database for thermal and electromagnetic parameters of biological tissues," version 2.4, July 30th, 2013 www.itis.ethz.ch/database.

[13] C. Gabriel, S. Gabriel, and E. Corthout, "The dielectric properties of biological tissues: I. literature survey,' Phys. Med. Biol., vol. 41, pp. 2231-2249, 1996

[14] Biotronik SE \& Co. KG, Linox ${ }^{\text {smart }}$ S. Tripolar ICD lead with active fixation, Technical Manual, 2014.

[15] G. Fröhlig, J. Carlsson, J. Jung, W. Koglek, B. Lemke, A. Markewitz, and J. Neuzner, Eds., Herzschrittmacher- und Defibrillator-Therapie. Indikation - Programmierung - Nachsorge. Stuttgart: Thieme, 2005 